\title{
IgA1 levels in milk and serum samples from intestinal parasite-infected or normal puerperae
}

\author{
Marianna Nascimento Manhani, Julia Maria Costa Cruz, Daniela Marques de Lima Mota Ferreira', \\ Vânia Olivetti Steffen Abdallah', Maria Aparecida de Souza/ ${ }^{+}$
}

Instituto de Ciências Biomédicas 'Departamento Pediatria, Universidade Federal de Uberlândia, Av. Pará 1720 - BI. 6T07, 38400-902 Uberlândia, MG, Brasil

In this study, IgA1 levels in the milk and serum of puerperae were compared and a correlation was established between the levels of this immunoglobulin and the occurrence of parasitism. Eighty-three paired milk and serum samples were obtained from puerperal and IgAl levels were analyzed. In addition, the presence of intestinal parasites in stool samples from these puerperae was determined. Twelve puerperae tested positive for intestinal parasites and all their samples presented an IgA1 ELISA Index $>1$. There was a correlation between serum and milk IgAI levels and puerperae with any parasite in their stool $(r=0.6723 ; p=0.0166)$. This finding may reinforce the importance of breast-feeding for the protection of neonates.

Keywords: IgA1 - breast-milk - puerperae - helminthes - intestinal parasites

Parasitic infections are characterized by the stimulation of immunologic mucosal mechanisms mediated by cells or antibodies (Else 2005). Immunoglobulin A (IgA) represents the major antibody class for defense against extracellular parasites at respiratory, gastrointestinal and genitourinary mucosa (Antonella et al. 2005). The main mechanism of protection against pathogen-derived antigens provided by mucosal immunity is through IgAproducing cells. IgA neutralizes and prevents the entry or establishment of potentially harmful antigens in the host (Senior \& Woof 2005). This antibody exists in two isotypes in humans: IgA1 and IgA2. In human serum, IgA1 represents $90 \%$ of the IgA composition (Yoo \& Morrison 2005) while IgA1 contributes from $70 \%-95 \%$ of the IgA composition in human maternal milk (Roitt et al. 2001).

In this way, human milk provides an efficient protection after birth by stopping the adhesion of infectious agents on the mucosal membranes, thereby preventing their entry into tissues of neonates (Hanson \& Korotkova 2002). Human neonates are born with an immature and naively-acquired immune system, and many of the innate components of mucosal immunity are not fully developed. Hence, the innate immune system of human milk is an important complement to the mucosal barrier of the developing gut (Newburg 2005).

Lectins bind specifically, reversibly, and non-covalently to carbohydrates, without altering its attached covalent structure (De-Simone et al. 2006). Jacalin is a lectin present in the seeds of the jackfruit (Artocarpus integrifolia) that binds specifically to human $\operatorname{IgA} 1$

Financial support: UFU

+Corresponding author: masouza@ufu.br

Received 8 April 2008

Accepted 21 July 2008
(Roque-Barreira \& Campos-Neto 1985). This lectin does not bind $\operatorname{Ig} \mathrm{A} 2$ due to a deletion of 13 amino acids and four acetylgalactosamin residues in the hinge region of this antibody isotype (Gregory et al. 1987). Thus, the aim of this study was to evaluate IgA1 levels in milk and serum from puerperae and whether the secretion of this immunoglobulin correlates with the presence of intestinal parasites.

Puerperae with normal pregnancies, hospitalized in the Hospital de Clínicas da Universidade Federal de Uberlândia (HC-UFU), were contacted for voluntary participation in the study, and a total of 83 accepted the invitation to participate. The Ethics Committee for Research of the UFU approved this study. Three stool samples were collected from each woman, one sample from each of three consecutive days, between the 15th and the 30th day postpartum (pp), totaling 249 samples. All samples were maintained in plastic containers without preservatives and kept at $4^{\circ} \mathrm{C}$ until the third sample was collected. The samples were then analyzed in order to detect intestinal parasites by the Baermann method and the Hoffman, Pons and Janer method. To certify the accuracy of each method, a triplicate sample was made, the sediment was stained with lugol and the sample was analyzed with a light microscope (100X and 400X magnification). In addition, serum samples from puerperae were available from the Gynecology and Obstetrics Service of the HC-UFU. Milk samples (approximately $10 \mathrm{ml}$ of foremilk) were manually collected in plastic vials by the puerperae themselves during the morning of the 15th and 30th day pp. These samples were centrifuged at $770 \mathrm{~g}$ $(2,500 \mathrm{rpm})$ at $4^{\circ} \mathrm{C}$ for $10 \mathrm{~min}$ (Sorvall RC 5C Plus, USA). The lipid layer was removed and discarded. All samples were stored at $-20^{\circ} \mathrm{C}$ until use. IgA1 levels in milk and serum were determined by a jacalin-capture enzyme-linked immunosorbent assay (ELISA), according to Gomes et al. (2006). Results were expressed as an ELISA Index (EI) and determined as follows: $\mathrm{EI}=$ absorbance of test sample/cut off, where the cut off was calculated as the mean value of blank absorbance plus three times the standard 
deviation; EI values $>1.0$ were considered positive for the presence of IgA1. The blank mean value was 0.091 and the standard deviation was 0.017 , resulting in a cut off of 0.142 .

Statistical analysis was performed based on the geometric mean (GM) and on the confidence interval (CI) of $95 \%$. Comparisons between the different GMs of IgA1 levels determined by ELISA were analyzed by the Mann Whitney test and the $t$ test with Welch's correction. The Pearson test procedure was adopted to test the correlation between the IgA1 levels in paired samples of human milk and serum. A p value $<0.05$ was considered to be statistically significant (GraphPad Prism version 4.0 Software, Inc).

The parasitological analysis indicated that $14.4 \%$ of the samples (12/83) were infected with at least one species of intestinal parasites. The protozoan Giardia intestinalis $(\mathrm{n}=1)$ as well as Strongyloides stercoralis $(\mathrm{n}=4)$, hookworms $(\mathrm{n}=3)$, Enterobius vermicularis $(\mathrm{n}=2)$, Taenia $\mathrm{sp} .(\mathrm{n}=1)$ and Hymenolepis nana $(\mathrm{n}=1)$ helminthes were found in stool samples. Among them, hookworms and S. stercoralis have pulmonary life cycles, while the others, including G. intestinalis, develop directly in the gut. The GM of IgA1 levels in milk samples from all puerperae $(\mathrm{n}=83)$ was 3.564 (CI: 3.177-3.998), with a maximal value equal to 9.007 and a minimum equal to 1.092 . In contrast, IgA1 levels in serum samples showed a GM of 2.798 (CI: 2.5603.057), varying from 9.063-1.254 (Fig. 1A). There was a statistically significant difference between IgA1 levels in the milk and serum samples from all assessed puerperae $(\mathrm{p}=0.0021)$. In puerperae without parasites $(\mathrm{n}=71)$, the GM of IgA1 levels in the milk was 3.501 (CI: 3.5134.541), with values ranging from 9.007-1.092. The GM of IgA1 levels in serum samples was 2.749 (CI: 2.673-3.267), with a maximum value of 7.563 and a minimum of 1.423 (Fig. 1B). This demonstrated that IgA1 levels in milk and serum samples were significantly different $(p=0.0046)$.

On the other hand, puerperae with parasites presented the highest geometric mean of IgA1 levels in both milk and serum. With regard to milk samples, the geometric mean of IgA1 levels was 3.962 (CI: 3.513-4.541), with a maximum value of 7.507 and a minimum of 2.049. The GM of IgA1 levels in serum samples was 3.105 (CI: 2.222-4.801), ranging from 9.063-1.254 (Fig. 1C). However, there was not a statistical difference when mean values of $\operatorname{IgA} 1$ in milk and serum samples were compared $(p=0.319)$. Moreover, neither milk nor serum samples showed significant differences when all samples were compared ( $p=0.8234 ; p=0.6692$, respectively). These findings may be due to the small number of puerperae included in the study. A positive correlation between IgA1 levels in milk and serum samples $(\mathrm{p}=0.0383$; $\mathrm{r}=0.2278$ ) was observed in all puerperae (Fig. 2A). Non-parasitic puerperae did not show a positive correlation between IgA1 levels in milk and serum in paired samples ( $p=0.2954 ; r=0.1259)$ (Fig. 2B). Nevertheless, puerperae with parasitism $(n=12)$ showed a statistically significant correlation between IgA1 levels in paired samples ( $p=0.0166 ; r=0.6723$ ) (Fig. 2 C). Therefore, an interrelation between the production of IgA1 in paired milk and serum samples was found in puerperae with intestinal parasites.

In addition, we detected IgA1 levels from breast milk and serum by using a jacalin-capture ELISA test. This method is fast, relatively easy test to perform and leads to the specific detection of IgA1 in biological samples. Other studies demonstrated the use of the jacalin test to not only detect IgA1 in serum and saliva samples by ELISA (Cullina \& Greally 1993, Gomes et al. 2006), but also to bind red cells (Green et al. 1993). Still, this is the first report in which the jacalin test was used in order to detect IgA1 levels in breast-milk samples.

Breast-feeding compensates for the relative immaturity of host defense mechanisms in neonates by providing considerable amounts of secretory $\operatorname{IgA}$ antibodies directed against the microbial flora of the mother and her environment. These antibodies bind microbes that appear on the infant's mucosal membranes, preventing
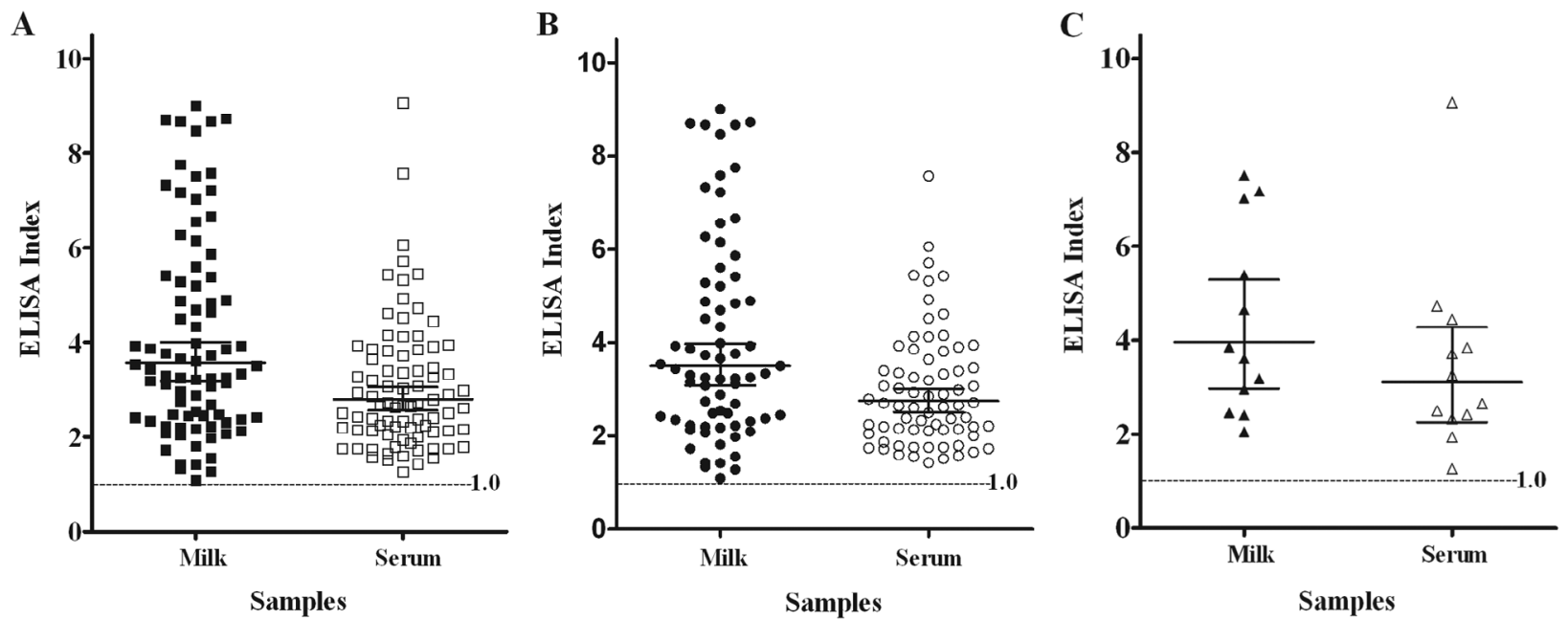

Fig. 1: geometric means of IgA1 levels in milk and serum samples, expressed as ELISA Index (EI) indicating maximum and minimum values and the confidence interval of geometric means. A: all puerperae $(n=83)$; B: puerperae without parasites $(n=71)$; C: puerperae with parasites $(n=12)$. EI $>1$. 
A

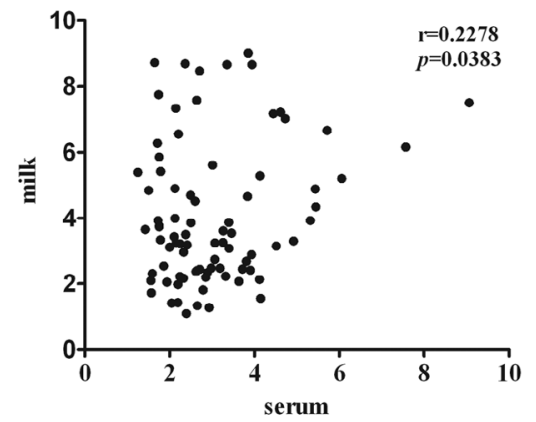

B

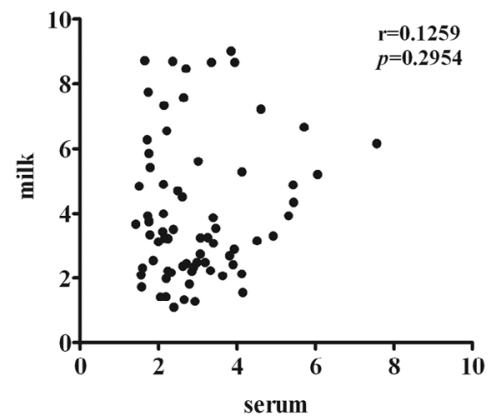

C

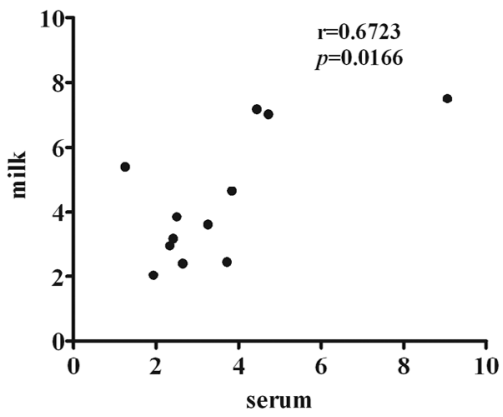

Fig. 2: correlation between IgA1 levels in milk and serum samples analyzed, expresses as ELISA index (EI). A: correlation of all puerperae samples $(n=83)$; B: correlation of samples from puerperae without parasites $(n=72)$; : correlation of samples from puerperae with parasites $(n=12)$.

activation of the pro-inflammatory defenses (Hanson 2007). In a child intestinal parasitism and breast-feeding study, Costa-Macedo and Rey (2000) suggested that natural maternal breast-feeding contributes to the control of enteric parasitosis in suckles. This protective role of breast milk is also suggested in our study because there was a positive correlation between $\operatorname{IgA} 1$ secretion in milk and serum samples of parasitic puerperae. Thus, $\operatorname{Ig} \mathrm{A}$ in milk is initially derived from the serum, but later is produced by plasma cells within the mammary tissue (Macpherson et al. 2001).

In this work, the correlation between the IgA secretion in milk and serum samples from parasitic puerperae suggests a connection between mucosal and serological immune systems in the response to parasites. The migration of lymphocytes from the lymphoid tissues of the intestines to mammary glands during late pregnancy contributes to this finding. This 'entero-mammaric link' of lymphocyte migration provides the milk with antibodies against those pathogens to which the mother was exposed previously (Lawrence \& Lawrence 2005). This connection was also hinted at in our study through IgA1 pairing among puerperae with intestinal parasites.

In conclusion, these findings suggest a positive correlation between IgA secretion in milk and serum samples from puerperae who are infected with parasites. This correlation may be explained by the mucosal stimulation that is caused by intestinal parasites. The composition of milk IgA is an important example of how remarkable systemic recirculation is following $\mathrm{B}$ cell induction in the intestine (Macpherson et al. 2001). Importantly, our data may reinforce the value of breast-feeding for the protection of neonates.

\section{ACKNOWLEDGMENTS}

To Daniel Stockdell, for language assistance, and Marcio L. De Paula, for critically reviewing this manuscript.

\section{REFERENCES}

Antonella B, Politis I, Pecorini C, Fusi E, Chronopoulou R, Dell'orto V 2005. Biological effects of milk proteins and their peptides with emphasis on those related to the gastrointestinal ecosystem. J Dairy Res 72: 66-72.

Costa-Macedo LM, Rey L 2000. Maternal and child intestinal parasitism and breast-feeding. Rev Soc Bra Med Trop 33: 371-375.
Cullina MJ, Greally JF 1993. A novel lectin-based enzyme-linked immunosorbent assay for the measurement of IgA1 in serum and secretory IgA1 in secretions. Clin Chim Acta 216: 23-38.

De-Simone SG, Netto CC, Silva Jr FP 2006. Simple affinity chromatographic procedure to purify-galactoside binding lectins. $J$ Chromatogr B 838: 135-138.

Else KJ 2005. Have gastrointestinal nematodes outwitted the immune system? Parasite Immunol 27: 407-415.

Gomes MAB, Rodrigues FH, Afonso-Cardoso SR, Buso AM, Silva AG, Favoreto-Jr S, Souza MA 2006. Levels of immunoglobulin A1 and messenger RNA for interferon $\mathrm{c}$ and tumor necrosis factor a in total saliva from patients with diabetes mellitus type 2 with chronic periodontal disease. J Periodont Res 41: 177-183.

Green F, Bubb MO, Conradie JD 1993. Use of jacalin as a solid phase in ABO reverse grouping. Vox Sang 65: 232-238.

Gregory RL, Rundegren J, Arnold RR 1987. Separation of human IgA1 and IgA2 using jacalin-agarose chromatography. J Immunol Methods 99: 101-106.

Hanson LA 2007. Session 1: Feeding and infant development breastfeeding and immune function. Proc Nutr Soc 66: 384-396.

Hanson LA, Korotkova M 2002. The role of breastfeeding in prevention of neonatal infection. Semin Neonatol 7: 275-281.

Lawrence RA, Lawrence RM 2005. Host-resistance factors and immunologic significance of human milk. In RA Lawrence, RM Lawrence, Breastfeeding: A guide for the medical profession, 6th ed., Mosby, MO, USA, p. 171-214.

Macpherson AJ, Hunziker L, McCoy K, Lamarre A 2001. IgA response in the intestinal mucosa against pathogenic and nonpathogenic microorganisms. Microbes Infec 3: 1021-1035.

Newburg DS 2005. Innate immunity and human milk. J Nutr 135: 1308-1312.

Roitt I, Brostoff J, Male D 2001. In L Crowe (ed.), Imunology, 6th ed., Harcourt Health Science Company, London, p. 75-96.

Roque-Barreira MC, Campos-Neto A 1985. Jacalin: an IgA-binding lectin. J Immunol 134: 1740-1743.

Senior BW, Woof JM 2005. Effect of mutations in the human immunoglobulin A1 (IgA1) hinge on its susceptibility to cleavage by diverse bacterial IgA1 proteases. Infect Immun 73: 1515-1522.

Yoo EM, Morrison SL 2005. IgA: an immune glycoprotein. Clin Immunol 116: 3-10. 